\title{
EFECTO DE TRES DIETAS COMERCIALES EN EL CRECIMIENTO Y SOBREVIVENCIA DE ALEVINOS DE PEZ ÁNGEL, Pterophyllum scalare (PERCIFORMES, CICHLIDAE) VARIEDAD MARMOLEADA
}

\author{
Liliana CERNA-MEZA ${ }^{1}$, Fred CHU-KOO ${ }^{2}$, Fernando ALCÁNTARA-BOCANEGRA ${ }^{3}$ y Luis MORI- \\ PINEDO \\ 1 Facultad de Ciencias Biológicas. Universidad Nacional de la Amazonía Peruana (UNAP) E-mail: licernam@gmail.com / \\ Imori49@hotmail.com \\ 2 Instituto de Investigaciones de la Amazonía Peruana. Filial Amazonas. E-mail: fchu@iiap.org.pe \\ 3 Instituto de Investigaciones de la Amazonía Peruana. Programa para el Uso y Conservación del Agua y sus Recursos - \\ AQUAREC.E-mail:fab_001@hotmail.com
}

\section{RESUMEN}

El objetivo del estudio fue evaluar el efecto de tres dietas comerciales en el crecimiento y la sobrevivencia de alevinos del pez ángel (Pterophyllum scalare), variedad marmoleada, provenientes de reproducción en cautiverio. El experimento se llevó a cabo en el Centro de Investigación y Promoción Popular (CENDIPP) de la ciudad de Iquitos. Se emplearon tres dietas comerciales para peces ornamentales, así como los siguientes tratamientos experimentales: $\mathrm{T} 1=$ Nutrafin Max $(43 \% \mathrm{~PB}$, en pellets), $\mathrm{T} 2=$ Tetramin $(47 \%$ de proteína bruta, en escamas) y T3 $=$ Sera ( $46,2 \% \mathrm{~PB}$, en escamas), que fueron asignados aleatoriamente por triplicado en nueve acuarios de $126 \mathrm{~L}$ de capacidad. Noventa peces, repartidos al azar en grupos de 10 individuos, fueron empleados para el estudio. Luego de un periodo de adaptación de 10 días, se inició la fase experimental, alimentando a los peces con dietas diarias correspondientes al 5\% de su biomasa corporal, dividiendo la ración dos veces al día durante 90 días. Al final del experimento, los peces alimentados con T2 lograron mejores resultados en ganancia de peso, tasa de crecimiento específico, conversión alimenticia y factor de condición $(\mathrm{P}<0,05)$, seguido de T3. No se registró diferencias en el porcentaje de sobrevivencia entre tratamientos $(\mathrm{P}>0,05)$.

PALABRAS CLAVE: Pterophyllum scalare, alimentación, dietas, crecimiento, sobrevivencia.

\section{EFFECTS OF THREE COMMERCIAL DIETS ON GROWTH AND SURVIVAL OF MARBLED ANGEL FISH Pterophyllum scalare (PERCIFORMES, CICHLIDAE) FINGERLINGS}

\begin{abstract}
The goal of this study was to evaluate the effects of three commercial diets on the growth and survival of marbled angel fish (Pterophyllum scalare) fingerlings obtained from local farmers. The experiment was carried out at the People Research and Promotion Center (CENDIPP) facilities, located nin Iquitos City. Three commercial diets used for ornamental fish were tested as experimental dietary treatments: $\mathrm{T} 1=$ Nutrafin Max ( $43 \% \mathrm{CP}$, as pellets), $\mathrm{T} 2=$ Tetramin ( $47 \%$ of crude protein, as flakes), and $\mathrm{T} 3=$ Sera $(46.2 \% \mathrm{CP}$, as flakes), which were randomly assigned by triplicate into nine $126-\mathrm{L}$ glass-made aquaria. Ninety fish randomly divided in groups of 10 individuals and distributed into the nine aquaria were used in the study. After an adaptation period of 10 days, fish were fed their respective diets at a feeding rate equivalent to $5 \%$ of fish biomass daily, twice per day during 90 days. At the end of the experiment, fish fed with $\mathrm{T} 2$ achieved best performance in weight gain, specific growth rate, feed conversion ratio and condition factor $(\mathrm{P}<0.05)$, followed by fish fed with T3. No significant differences were found for survival rate among treatments $(\mathrm{P}>0.05)$.
\end{abstract}

KEYWORDS: Pterophyllum scalare, feeding, diets, growth, survival. 


\section{INTRODUCCIÓN}

La diversidad ictiológica presente en los cuerpos de agua de la Amazonía peruana es considerada superior a otras cuencas del país, estimándose en unas 726 especies, siendo utilizadas aproximadamente 70 de ellas para el consumo humano directo, mientras que otras 420 son consideradas como recurso ornamental (Ortiz \& Iannacone, 2008).

En un estudio reciente, García et al. (2011), consideran que la cuenca del Ucayali es la más importante para la pesquería ornamental en la región Loreto, aportando el $41 \%$ del volumen de captura, seguido por los ríos Nanay, con $25 \%$, Napo, con $10 \%$, Itaya y Amazonas, con $9 \%$, y Putumayo con el $4 \%$. Según estos autores, entre los años 2000 y 2010 los Siluriformes fueron los peces más representativos, con el $57 \%$ de las capturas para fines ornamentales, seguidos de los Characiformes, con el $26 \%$ y los Osteoglossiformes, con el $13 \%$ (García et al., 2011).

En piscicultura, la alimentación, una vez finalizada la absorción del vitelo, es uno de los factores más importantes en la crianza de peces (Garvía, 2009). Los alimentos inertes como la yema de huevo en suspensión, el plancton natural, Moina y Tubifex son los productos comúnmente más utilizados (Lim et al., 2003).

La fabricación de alimentos balanceados ha ido evolucionando notablemente, de manera que el color, la textura, el olor, el sabor, la forma y la flotabilidad del alimento han sido estudiadas para lograr que el pez lo consuma eficazmente, ya sea por ser llamativo en color y forma, o por la similitud al alimento consumido en el medio natural o en criaderos (Garvía, 2009).

Al ser la alimentación un factor importante durante el manejo y producción de peces ornamentales, el objetivo de la presente investigación fue evaluar el efecto de tres dietas balanceadas comerciales en el crecimiento y sobrevivencia de alevinos de Pterophyllum scalare "pezángel”, variedad marmoleada.

\section{MATERIALES Y MÉTODOS Área de estudio}

El estudio se llevó a cabo en las instalaciones acuícolas de la organización no gubernamental conocida como Centro de Investigación y Promoción Popular (CENDIPP), ubicada en la Calle Moore $N^{a} 154$ de la ciudad de Iquitos, Provincia de Maynas, Departamento de Loreto.

\section{Procedencia de los peces}

Para el presente trabajo, fueron empleados un total de 90 alevinos de Pterophyllum scalare de la variedad marmoleada, producidos en cautiverio dentro de las instalaciones acuícolas de la ONG CENDIPP. El peso y longitud promedio inicial de estos peces fue de $0,52 \pm 0,08 \mathrm{~g}$ y $2,7 \pm 0,11 \mathrm{~cm}$, respectivamente.

\section{Unidades experimentales}

Se utilizaron nueve (09) acuarios de vidrio con un volumen de $126 \mathrm{~L}$. Las dimensiones de los acuarios fueron: $37 \mathrm{~cm}$. de alto; $38 \mathrm{~cm}$. de ancho y 88 $\mathrm{cm}$. de largo, todos ellos equipados con bombas de aire y filtros de esponja.

\section{Diseño experimental}

Los tres tratamientos (dietas comerciales) seleccionados para el estudio, fueron aleatoriamente asignados a sus respectivos acuarios por triplicado, siguiendo un diseño completamente al azar, distribuyéndose los 90 peces en grupos de 10 peces/acuario (Tabla 1).

Las tres dietas comerciales empleadas como tratamientos pertenecen a marcas reconocidas en el mercado de la acuariofilia. La primera dieta es fabricada por HAGEN (Nutrafin Max), la segunda por TETRA (Tetramin), y la última por la empresa SERA. En la Tabla 2, se presentan los valores nutricionales de cada una de ellas.

\section{Alimentación de los peces}

Los peces fueron alimentados con una tasa equivalente al 5\% de la biomasa presente en cada acuario, determinada para cada uno de ellos al inicio del experimento y en cada muestreo biométrico quincenal.

La alimentación se realizó dos veces por día (09:00 y 17:00 h); los siete días de la semana, durante los 90 días que duró la ejecución de la fase experimental. Se trabajó con fotoperiodo de 12:12 (Doce horas luz; doce horas oscuridad).

\section{Tratamiento del agua}

El agua utilizada provino de la red pública de agua potable. El agua era previamente almacenada en un tanque de polietileno con capacidad de $2500 \mathrm{~L}$ para ser pre-tratada a través de un filtro artesanal constituido por una capa mediana de perlón, bioesponja, cerámica para biofiltro, carbón activado, piedra antimonio y piedra anti-fosfato, antes de su uso en el experimento. El agua era filtrada por un 
periodo de 24 a $36 \mathrm{~h}$ para luego ser trasvasada a un tanque de $1000 \mathrm{~L}$, que finalmente abastecía a los acuarios de vidrio.

\section{Índices zootécnicos}

Para verificar el comportamiento de los tres parámetros experimentales considerados en el presente proyecto (crecimiento, asimilación de los alimentos y sobrevivencia de los peces), se evaluaron los siguientes parámetros: Ganancia de peso (GP), Ganancia de longitud (GL), Tasa de Crecimiento Específico (TCE), Ganancia de Biomasa (GB), Índice de Conversión Alimenticia Aparente (ICAA), Factor de Condición (K) y la Tasa de Sobrevivencia $(\mathrm{S})$.

\section{Calidad de agua}

Se realizaron monitoreos diarios del oxígeno disuelto, $\mathrm{pH}$ y temperatura del agua con un Oxímetro YSI Modelo 556 (YSI Instrument Co., Ohio, USA). La medición de los niveles de amonio, nitrito y nitrato, se realizaron quincenalmente con un Kit de la empresa Hach.

\section{Análisis de datos}

Los datos obtenidos de los muestreos fueron almacenados y procesados en hojas de cálculo Excel para luego ser evaluados a través de análisis de varianza simple (nivel de confianza del 95\%), considerando que se cumplan previamente todas las presunciones básicas del ANOVA. Se aplicó la prueba de comparación de promedios de TukeyKramer $(\alpha=0,05)$ cuando se encontraron diferencias significativas en el ANOVA. Se utilizó el programa estadístico JMP IN® Versión 4.0.4. (SAS Institute, USA) como herramienta para el análisis. Los resultados de crecimiento e índices zootécnicos son mostrados como el promedio \pm desviación estándar de la media. Los datos expresados en porcentajes fueron transformados por el método del arcoseno previo a su análisis por ANOVA.

\section{RESULTADOS}

\section{Crecimiento de los peces}

El crecimiento mayor, tanto en peso como en longitud total, se registró en los peces alimentados con la dieta $\mathrm{T} 2$, seguida de aquellos alimentados con $\mathrm{T} 3$, mientras que los ejemplares que recibieron la dieta T1 mostraron el desempeño más bajo en términos de crecimiento en peso y longitud durante el experimento (Tabla 3).

Como consecuencia de los tratamientos mostrados en la Tabla 3, se obtiene que los mejores resultados, en términos de ganancia de peso, tasa de crecimiento específico y factor de condición, se lograron en peces alimentados con la dieta Tetramin $(\mathrm{P}<0,05)$, seguida de los peces alimentados con Sera. Los peces alimentados con la dieta Nutrafin Max, fueron los que presentaron menor ganancia de peso, TCE y factor de condición (Tabla 4).

Según la prueba de Tukey, los peces alimentados con las dietas Tetramin y Sera presentaron similares niveles de conversión alimenticia $(\mathrm{P}>0,05)$, en cambio, la dieta Nutrafin Max fue poco aprovechada por los peces $(\mathrm{ICAA}=3,82)$. El porcentaje de sobrevivencia no presentó diferencia significativas entre tratamientos $(\mathrm{P}>0,05)$, obteniéndose al final del experimento un promedio de $96,67 \%$.

\section{Calidad de agua}

Los valores de temperatura, $\mathrm{pH}$ y el oxígeno disuelto, se mantuvieron dentro de los valores adecuados para el cultivo de peces, siendo los compuestos nitrogenados los únicos que se mantuvieron ligeramente por encima de los niveles recomendados para la acuicultura de especies amazónicas.

Tabla 1. Resumen del diseño experimental del estudio

\begin{tabular}{cccc}
\hline $\begin{array}{c}\text { Tratamiento } \\
\text { Dieta }\end{array}$ & \# Peces & Réplicas & $\begin{array}{c}\text { \# Total de } \\
\text { Peces }\end{array}$ \\
\hline T1 & 10 & 3 & 30 \\
T2 & 10 & 3 & 30 \\
T3 & 10 & 3 & 30 \\
\hline & Total & & 90 \\
\hline
\end{tabular}


Tabla 2. Composición nutricional de las dietas comerciales empleadas como tratamientos experimentales en el presente estudio.

\begin{tabular}{lccc}
\hline & $\begin{array}{c}\text { T1 } \\
\text { Nutrafin Max }\end{array}$ & $\begin{array}{c}\text { T2 } \\
\text { Tetramin }\end{array}$ & $\begin{array}{c}\text { T3 } \\
\text { Sera }\end{array}$ \\
\hline Presentación & Pellet & Hojuela & Hojuela \\
\hline Proteína (\%) & 43,0 & 47,0 & 46,2 \\
\hline Grasa (\%) & 4,0 & 10,0 & 8,9 \\
\hline Fibra (\%) & 3,0 & 3,0 & 2,3 \\
\hline Humedad (\%) & 8,0 & 6,0 & 6,7 \\
Ceniza (\%) & 9,0 & 9,0 & 11,9 \\
\hline Carbohidratos (\%) & 33,0 & 25,0 & 24,0 \\
Energía Bruta (Kcal/Kg) & 3830 & 4250 & 4070 \\
\hline
\end{tabular}

Tabla 3. Peso y longitud inicial y final (promedio \pm desviación estándar) obtenidos en alevinos del pez ángel (Pterophyllum scalare), alimentados con tres dietas comerciales durante 90 días.

\begin{tabular}{ccccc}
\hline \multirow{2}{*}{ Parámetros } & \multicolumn{3}{c}{ TRATAMIENTOS } & \multirow{2}{*}{$\boldsymbol{P}$} \\
\cline { 2 - 4 } & T1 & T2 & T3 & \\
\hline Li & $2,63 \pm 0,08$ & $2,75 \pm 0,12$ & $2,74 \pm 0,14$ & 0,4278 \\
LF & $3,99 \pm 0,31^{\mathrm{b}}$ & $5,83 \pm 0,07^{\mathrm{a}}$ & $5,49 \pm 0,19^{\mathrm{a}}$ & 0,0001 \\
$\mathbf{P i}$ & $0,47 \pm 0,05$ & $0,5 \pm 0,1$ & $0,6 \pm 0,1$ & 0,1106 \\
PF & $1,33 \pm 0,32^{\mathrm{c}}$ & $5,17 \pm 0,32^{\mathrm{a}}$ & $3,93 \pm 0,40^{\mathrm{b}}$ & $<0,0001$ \\
\hline
\end{tabular}

Leyenda: Li: Longitud inicial, LF: Longitud final, Pi: Peso inicial, PF: Peso final

Tabla 4. Índices zootécnicos (promedio \pm desviación estándar) obtenidos en la crianza de alevinos de Pterophyllum scalare, alimentados con tres dietas comerciales durante 90 días.

\begin{tabular}{ccccc}
\hline \multirow{2}{*}{ Parámetros } & \multicolumn{3}{c}{ TRATAMIENTOS } & \multirow{2}{*}{$\boldsymbol{P}$} \\
\cline { 2 - 4 } & T1 & T2 & T3 & \\
\hline TCE & $1,15 \pm 0,24^{\mathrm{c}}$ & $2,59 \pm 0,07^{\mathrm{a}}$ & $2,09 \pm 0,13^{\mathrm{b}}$ & 0,0001 \\
GP & $0,87 \pm 0,30^{\mathrm{c}}$ & $4,67 \pm 0,32^{\mathrm{a}}$ & $3,33 \pm 0,35^{\mathrm{b}}$ & $<0,0001$ \\
ICAA & $3,82 \pm 0,94^{\mathrm{a}}$ & $1,5 \pm 0,08^{\mathrm{b}}$ & $1,91 \pm 0,09^{\mathrm{b}}$ & 0,003 \\
K & $2,06 \pm 0,09^{\mathrm{c}}$ & $2,61 \pm 0,09^{\mathrm{a}}$ & $2,36 \pm 0,06^{\mathrm{b}}$ & 0,0008 \\
S\% & $96,67 \pm 5,77$ & 100 & 100 & 0,4219 \\
\hline
\end{tabular}

Leyenda: TCE: Tasa de Crecimiento Específico, GP: Ganancia de Peso, ICAA: Índice de Conversión Alimenticia Aparente, K: Factor de Condición, S\%: Tasa de Sobrevivencia. 
Tabla 5. Valores de los parámetros de calidad de agua (promedio \pm desviación estándar) registrados en la crianza de alevinos de Pterophyllum scalare, variedad marmoleado, alimentados con tres dietas comerciales durante 90 días.

\begin{tabular}{lccc}
\hline \multirow{2}{*}{ Parámetros } & \multicolumn{3}{c}{ TRATAMIENTOS } \\
\cline { 2 - 4 } & T1 & T2 & T3 \\
\hline Temperatura $\left({ }^{\circ} \mathbf{C}\right)$ & $25,16 \pm 0,65$ & $25,42 \pm 0,57$ & $24,99 \pm 0,59$ \\
pH & $6,28 \pm 0,3$ & $6,14 \pm 0,28$ & $6,18 \pm 0,31$ \\
Oxígeno $(\mathbf{m g} / \mathrm{l})$ & $5,90 \pm 1,04$ & $6,04 \pm 0,89$ & $6,13 \pm 0,77$ \\
Amonio & $1,41 \pm 10,1$ & 1 & 0,5 \\
Nitrito & $7 \pm 0,16$ & $0,3 \pm 0,12$ & $0,33 \pm 0,19$ \\
Nitrato & $0,85 \pm 2,03$ & $3,6 \pm 4,95$ & $3,43 \pm 5,08$ \\
\hline
\end{tabular}

\section{DISCUSIÓN}

\section{Crecimiento de los peces}

El presente estudio ha mostrado que los mejores niveles de crecimiento (peso final, longitud final, ganancia de peso, factor de condición, índice de conversión alimenticia y tasa de crecimiento específico) fueron logrados con la dieta Tetramin, seguida de Sera, siendo el menor desempeño productivo observado en aquellos peces alimentados con la dieta Nutrafin Max.

La superioridad mostrada por las dos primeras dietas, puede hipotéticamente deberse a varios factores. Entre ellos podemos citar a los siguientes: i) su mayor contenido proteico, grasa y energía, ii) el tipo de presentación de las dietas, y consecuentemente, iii) su mejor asimilación por los peces.

Al referirnos al mayor contenido proteico, existen varios estudios que apoyan esta suposición. Por ejemplo, Kruger et al. (2001), evaluó el efecto de diferentes niveles de proteína en la dieta del pez espada (Xiphophorus helleri) y verificó que los ejemplares en experimentación presentaron mayores niveles de crecimiento cuando fueron alimentados con las dietas de mayor contenido proteico. Por su parte, Kim et al. (2005), realizó un estudio similar en el lenguado (Paralichthys olivaceus), encontrando los mismos resultados. Otro ejemplo reciente es el estudio publicado por Del Risco et al. (2008), donde los autores evaluaron el crecimiento de alevinos de paiche (Arapaima gigas) alimentados con raciones conteniendo tres niveles de proteína dietaria $(35,40$ y $45 \%$ PB) y determinaron que la ganancia de peso y la tasa de crecimiento específico fue evidentemente superior en los peces alimentados con las dietas que contenían 40 y $45 \%$ de proteína, en relación a los peces alimentados con la ración que poseía solo $35 \%$. Ejemplos similares son reportados para otras especies de peces como Sander lucioperca (Schulz et al., 2007), Pagrus pagrus (Schuchardt et al., 2008) y Piaractus brachypomus (Vásquez-Torres et al., 2011).

Las proteínas son utilizadas para el crecimiento y como fuente principal de energía, luego de las grasas (Tacon \& Cowey, 1987). Varios estudios determinaron que $P$. scalare solamente requiere entre $26-34 \%$ de PB para un buen desempeño (Zuanon et al., 2006; Ribeiro et al., 2007); por tanto, las dietas utilizadas en el presente estudio superaban ampliamente los requerimientos nutricionales de la especie. En tal sentido, los resultados obtenidos en el presente trabajo no se explican únicamente por el contenido proteico de las dietas; entrando a tallar entonces otros dos factores, como son el tipo de presentación y la mejor asimilación de las mismas.

Con relación al tipo de presentación de las dietas, cabe indicar que, según el proceso de elaboración de una dieta inerte para peces, ésta puede ser más atractiva, estable, de mejor textura, palatable y altamente digerible en los organismos que la consuman. En tal sentido, es necesario mencionar que las dos dietas que produjeron los mejores 
resultados en términos de crecimiento (Tetramin y Sera) fueron elaboradas como hojuelas (también llamada "escamas"), a través de un proceso de cocción similar a los alimentos extrusados, que les otorga una consistencia más blanda, coloración vistosa, mayor tiempo de flotabilidad y con una mejora notoria en la digestibilidad de los insumos que en conjunto componen la dieta (Davis \& Arnold, 1995). En el experimento, estas dos dietas fueron rápidamente consumidas por los alevinos a diferencia de la dieta peletizada (Nutrafin Max) que era menos palatable, tenía una capacidad de flotación limitada y su diámetro era superior al tamaño de la boca de los alevinos. Estos factores influyeron negativamente en la ingesta de la dieta, por lo que se optó por triturarlos antes de ser suministrados a los peces, lo que a la larga, constituyó una seria limitante para el crecimiento de los ejemplares alimentados con esta ración, siendo no adecuada para esta especie que se alimenta en los estratos medio y superficial de la columna de agua.

Los valores de TCE reportados en el presente estudio, son similares a los obtenidos por Takashi et al. (2010), quienes trabajando con tres dietas (Artemia, escamas y ración en polvo) registraron valores de 1,$83 ; 1,88 ; 2,72$, respectivamente en esta misma especie. Por su parte, Zuanon et al. (2006), registran valores mínimos de 2,31 y máximos de 2,61 , utilizando dietas en escamas con diferentes niveles proteicos $(34-46 \%)$. Estos resultados indican una relación directamente proporcional entre nivel proteico y TCE.

Respecto a la conversión alimenticia hubo notables diferencias cuando comparamos el ICAA obtenido para el T1 $(3,82)$ con los ICAA registrados para los peces del T2 y T3 $(1,5$ y 1,91 , respectivamente) indicando este resultado que hubo un mayor aprovechamiento de nutrientes en las dietas presentadas en la forma de escamas.

Autores como Luna-Figueroa (2000); Mañón (2008) y Soriano \& Hernández (2002), señalan que $P$. scalare presenta un desempeño productivo superior cuando es alimentado con Daphnia pulex $(52 \%$ de $\mathrm{PB})$, en comparación a alimentos balanceados como Wardley ( $45 \%$ de PB), Tetra-Bits (49\% de PB) y Sera (46\% de PB). Entretanto, Jiménez-Rojas et al. (2012) y Koca et al. (2009), manifiestan que al combinar dietas inertes con alimento vivo se mejora ostensiblemente el rendimiento productivo de esta especie.

Teniendo en cuenta los datos obtenidos en el presente estudio se puede afirmar que con el uso de dietas balanceadas se pueden lograr niveles de crecimiento y asimilación de nutrientes similares o incluso superiores a los obtenidos con alimento vivo.
Finalmente, el uso de dietas balanceadas con diferentes porcentajes proteicos y tipos de presentación, no influyó negativamente en la sobrevivencia de los alevinos, siendo siempre superior al 95\%. Valores similares fueron obtenidos por otros autores al trabajar con esta misma especie y utilizando dietas comerciales (Ribeiro et al., 2007; Takashi et al., 2008, Avendaño, 2008 y Koca et al., 2009).

\section{Calidad de agua}

En el presente estudio, los valores registrados de temperatura, $\mathrm{pH}$ y oxígeno disuelto se mantuvieron dentro de los rangos deseables para el buen desenvolvimiento de P. scalare (Pérez et al. 2003). Con relación a las concentraciones de compuestos nitrogenados, se registraron valores altos, sobre todo, en los acuarios donde se proporcionó la dieta peletizada, por lo que la presencia de estos compuestos fue debida a la descomposición del alimento no consumido. Ribeiro et al. (2007), indican que niveles de proteína por encima del óptimo exigido, determinan la desaminación de la proteína con un excesivo catabolismo proteico, causando aumento de la excreción branquial de nitrógeno amoniacal y la acumulación de compuestos nitrogenados en el agua.

\section{CONCLUSIONES}

Las dietas que produjeron el mejor desempeño en la crianza de alevinos de Pterophyllum scalare, en términos de crecimiento y conversión alimenticia, fueron en orden de importancia: la dieta Tetramin Hojuela ( $47 \%$ de PB), seguida de la dieta Sera Hojuela (46,2\%). La dieta Nutrafin Max Pellet $(43 \%)$ se presenta como la ración de menor efectividad en la promoción del crecimiento. Todas las dietas tuvieron un efecto positivo en la sobrevivencia final, siendo superior al $95 \%$ en todos los acuarios.

\section{REFERENCIAS BIBLIOGRÁFICAS}

Avendaño, L. 2008. Níveis de arraçoamento e freqüencia alimentar no desempenho produtivo do Acará-Bandeira. Universidade Estadual Paulista, Centro de Aqüicultura. São Paulo. Pp: $1-49$.

Davis, A., Arnold, D. 1995. Effects of two extrusion processing conditions on the digestibility of four cereal grains for Penaeus vannamei. Aquaculture, 133:287-294. 
Del Risco, M.; Velásquez, J.; Sandoval, M.; Padilla, P.; Mori, L.; Chu-Koo, F. 2008. Efecto de tres niveles de proteína dietaria en el crecimiento de juveniles de Paiche, Arapaima gigas (shinz, 1822). Folia Amazónica, 17(1-2): 29 - 37

García, A.; Vargas, G.; Sánchez, H.; Duponchelle, F.; Tello, S. 2011. Situación actual de la pesquería ornamental en la región Loreto-Perú entre los años 2000 y 2010. In: Agudelo, C.E., Duponchelle, F. (eds.). Comunicaciones del III Coloquio de la Red de Investigación sobre la Ictiofauna Amazónica-RIIA. p.46-51.

Garvía A. 2009. Nutrición y salud de los peces de acuario. [Online] URL: www.acuario profesional.com/pdf/NUTRICION_Y_SALUD EN LOS PECES DE ACUARIO.pdf. Acceso: 15/02/2011.

Jiménez-Rojas, J.; Alméciga-Díaz, P.; HerazoDuarte, D. 2012. Desempeño de juveniles del pez ángel Pterophyllum scalare alimentados con el oligoqueto Enchytraeus buchholzi. Universitas Scientiarum, 17(1): 28-34.

Kim, K.W.; Kang, Y.J.; Choi, S.M.; Wang, X.; Choi, Y.H.; Bai, S.; Jo, J.-Y.; Lee, J.H. 2005. Optimum dietary protein levels and protein to energy ratios in Olive Flounder Paralichthys olivaceus. Journal of the World Aquaculture Society, 36(2): 165-178.

Koca, S.; Diler, I.; Dulluc, A.; Yigit, N.; Bayrak, H. 2009. Effect of different feed types on growth and feed conversation ratio of Angel Fish Pterophyllum scalare Lichtenstein, 1823). Journal of Applied Biological Sciences, 3(2):610.

Kruger, D.P.; Britz, P.J.; Sales, J. 2001. Influence of varying dietary protein content at three lipid concentrations on growth characteristics of juvenile swordtails (Xiphophorus helleri Heckel, 1848). Aquarium Sciences and Conservation, 3: 275-280.

Lim, L. C.; Dhert, P.; Sorgeloos, P. 2003. Recent developments in the application of live feeds in the freshwater ornamental fish culture. Aquaculture, 227:319-331.

Luna - Figueroa, J.; Figueroa, J.; Hernández, L. 2000. Efecto de alimentos con diferente contenido proteico en la reproducción de pez ángel Pterophyllum scalare variedad perlada (Pisces: Cichlidae). Revista Ciencia y Mar, 4(11): 3- 10

Mañón, C. 2008. Reproducción del pez ángel Pterophyllum scalare (Pisces: Cichlidae) Lichtenstein, 1823. XIX Congreso de Investigación CUAM 2008. [Online]
URL:http:/www.acmor.org.mx/cuam/2008/224 -angel.pdf. Acceso: 19/02/2011.

Ortiz, N., Iannacone, J. 2008. Estado actual de los peces ornamentales amazónicos del Perú que presentan mayor demanda de exportación. Biologist, 6(1): 54-67.

Pérez, E.; Díaz, F.; Espina, S. 2003. Thermoregulatory behavior and critical thermal limits of the angelfish Pterophyllum scalare (Lichtenstein) (Pisces:Cichlidae). Journal of Thermal Biology, 28(8):531-537.

Ribeiro, F.; Rodrigues, L.; Fernandes, J. 2007. Desempenho de juvenís de Acará-Bandeira (Pterophyllum scalare) com diferentes níveis de proteína bruta na dieta. Boletin do Instituto de Pesca, São Paulo, 33(2): 195-203.

Santos, E.; Takashi, L.; Saita, M.; Da Silva, T.; Rigobelo, E. 2008. Efeito de diferentes alimentos na qualidade de agua na produçäo do Acará-Bandeira (Pterophyllum scalare). IV Simpósio de Ciências da UNESP - Dracena. V Encontro de Zootecnia-Unesp Dracena. 4p.

Schuchardt, D.; Vergara, J.M.; Fernandez-Palacios, H.; Kalinowski, C.T.; Hernandez-Cruz, C.M.; Izquierdo, M.S.; Robaina, L. 2008. Effects of different dietary protein and lipid levels on growth, feed utilization and body composition of red porgy (Pagrus pagrus) fingerlings. Aquaculture Nutrition, 14:1-9.

Schulz, C.; Bohm, M.; Wirth, M.; Rennert, B. 2007. Effect of dietary protein on growth, feed conversion, body composition and survival of pike perch fingerlings (Sander lucioperca). Aquaculture Nutrition, 13:373-380.

Soriano, M., Hernández, D. 2002. Tasa de crecimiento del Pez Ángel Pterophyllum scalare (Perciformes: Cichlidae) en condiciones de laboratorio. México. Acta Universitaria, 12(2): $28-33$.

Tacon, A., Cowey, C.B. 1987. The nutrition and feeding of farmed fish and farmed fish and shrimp-a training manual. 1. The essential nutrients. FAO. Trust Fund GCO/RLA/075/ITA. Brasilia, Brasil. 117 pp

Takashi, L.; Da Silva, T.; Fernandes, T.; Biller, J.; De Sandre, L. 2010. Efeito do tipo de alimento do desempenho produtivo de juvenis de acarábandeira (Pterophyllum scalare). Boletin do Institutio de Pesca São Paulo, 36(1): 1 - 8.

Vásquez-Torres, W.; Pereira-Filho, M.; AriasCastellanos, J. 2011. Optimum dietary crude protein requirement for juvenile cachama Piaractus brachypomus. Ciência Rural, 41(12): 2183-2189. 
Zuanon, J.; Salaro, A.; Balbino, E.; Saraiva, A.; Quadros, M.; Lima, R. 2006. Níveis de proteína bruta em dietas para alevinos de acará-bandeira Pterophyllum scalare. Revista Brasileira Zootecnia, 35(5): 1893-1896.
Recibido: 03/02/2014

Aceptado para publicación: 02/05/2014 